\title{
Static Buckling Analysis of a Quadratic-Cubic Model Structure Using the Phase Plane Method and Method of Asymptotics
}

\author{
A. C. Osuji ${ }^{1, *}$, A. M. Ette ${ }^{2}$ and J. U. Chukwuchekwa ${ }^{3}$ \\ ${ }^{1}$ Department of Mathematics, Federal University of Technology, Owerri, Imo State, Nigeria \\ e-mail: aatac2000@gmail.com \\ ${ }^{2}$ Department of Mathematics, Federal University of Technology, Owerri, Imo State, Nigeria \\ e-mail: tonimonsette@yahoo.com \\ ${ }^{3}$ Department of Mathematics, Federal University of Technology, Owerri, Imo State, Nigeria \\ e-mail: joyuchekwa@gmail.com
}

\begin{abstract}
The exact and asymptotic analyses of the buckling of a quadratic-cubic model structure subjected to static loading are discussed. The governing equation is first solved using the phase plane method and next, using the method of asymptotics. In the asymptotic method, we discuss the possibilities of using regular perturbation method in asymptotic expansions of the relevant variables to get an approximate analytical solution to the problem. Finally, the two results are compared using numerical results obtained with the aid of Q-Basic codes. In the two methods discussed in this work, it is clearly seen that the static buckling loads decrease as the imperfection parameters increase. It is also observed that the static buckling loads obtained using the exact method are higher than those obtained using the method of asymptotics.
\end{abstract}

\section{Introduction}

Buckling of elastic materials is a form of instability that occurs suddenly with large changes in deformation but little change in loading [1]. For this reason, it is a dangerous phenomenon that must be avoided in structural design because it can lead to catastrophic failure. Buckling of elastic structures can occur under static or dynamic loading conditions. Many serious research works have been done in both dynamic and static

Received: May 18, 2021; Accepted: July 2, 2021

2010 Mathematics Subject Classification: 00A69.

Keywords and phrases: static buckling, asymptotic and perturbation methods, phase plane method, elastic materials. 
buckling in recent times. Kołakowski [2] studied static and dynamic interactive buckling of composite columns, while Chitra and Priyadarsini [3] considered dynamic buckling of composite cylindrical shells subjected to Axial Impulse using finite element method. In the same token, the dynamic stability of suddenly loaded laminated cylindrical shells and the effect of static preloading upon the dynamic critical load were studied by Simitses [4] while Tabiei et al. [5] studied the numerical simulation of cylindrical laminated shells under impulsive lateral pressure and Tanov et al. [6] likewise discussed the effect of static preloading on dynamic buckling of laminated cylinders under sudden pressure. Jabareen and Izhak [7] on the other hand discussed the dynamic buckling of a beam on a nonlinear elastic foundation under step loading. Jankowski [8] discussed the static buckling of composite column-beams. The following review papers also outline recent research works in buckling: Patil et al. [9], Qatu [10] and Touati et al. [11]. Other investigations include Simitses [12] and Sahu and Datta [13].

Another form of loading proposed by Simitses [4] is known as the quasi-static loading and is such that the static pressure is applied gradually, at small enough rate so as to keep the inertial effects negligible, thus emulating a static analysis (see for example, Jeong [14], Russell et al. [15] and Zareiforoush et al. [16]), only to be trapped by a dynamic load after the initial static load.

We remark that buckling phenomena have been investigated analytically, numerically and experimentally for decades. However, most buckling problems in the literatures are investigated using numerical methods. Examples of such investigations include Touati et al. [11] and Eglitis et al. [17]. We must note that Lu and Wang [18] discussed the asymptotic solutions for buckling delamination induced crack propagation in the thin film-compliant substrate system while Lewandowski [19] studied the analysis of strongly non-linear free vibrations of beams using perturbation method. Similarly, Reboux et al. [20] studied the asymptotic analysis of the buckling of a highly shear-resistant vesicle while Eirik et al. [21] considered a semi-analytical model for global buckling and postbuckling analysis of stiffened panels. Much earlier on, Amazigo et al. [22] had studied asymptotic analysis of the buckling of imperfect columns on a nonlinear elastic foundation while Qiang et al. [23] considered the asymptotic solution of a dynamic buckling problem in elastic columns. Ette [24], on the other hand, used asymptotic expansions to examine the dynamic buckling of a spherical shell under an axial impulse while Ette [25] similarly used analytical methods to study a two-parameter dynamic buckling of a lightly damped spherical cap trapped by a step load. Ette and Onwuchekwa 
[26] also studied the static buckling of an externally pressurized finite circular cylindrical shell using asymptotic methods.

In this paper, the phase plane method and regular perturbation method in asymptotic expansions of the relevant variables (Bender and Orszag [27]) are used to analyze the buckling of a quadratic-cubic model structure under a static loading. Finally, numerical calculations are obtained with the help of Q-Basic codes and thereafter, beneficial conclusions are made. This simple quadratic elastic model structure was initially studied by Budiansky [28] while Danielson [29] made a refinement or an improvement on this initial model structure. In the same token, Ette [30] and Chukwuchekwa and Ette [31] similarly studied the same structure. We strongly believe that this simple model structure provides a generalization of some of the structures encountered in real physical structural materials.

\section{Formulation of the Problem}

The real essence of the simple model structure under investigation is ably captured by the spring arrangements as in Figure 1, which was first studied by Hutchinson and Budiansky [32]. Danielson [29] made an additional improvement on it by introducing an additional mass, $M_{0}$ and a spring with spring constant $K_{0}$, all with the aim of producing a pre-static buckling displacement, $\xi_{0}(T)$.

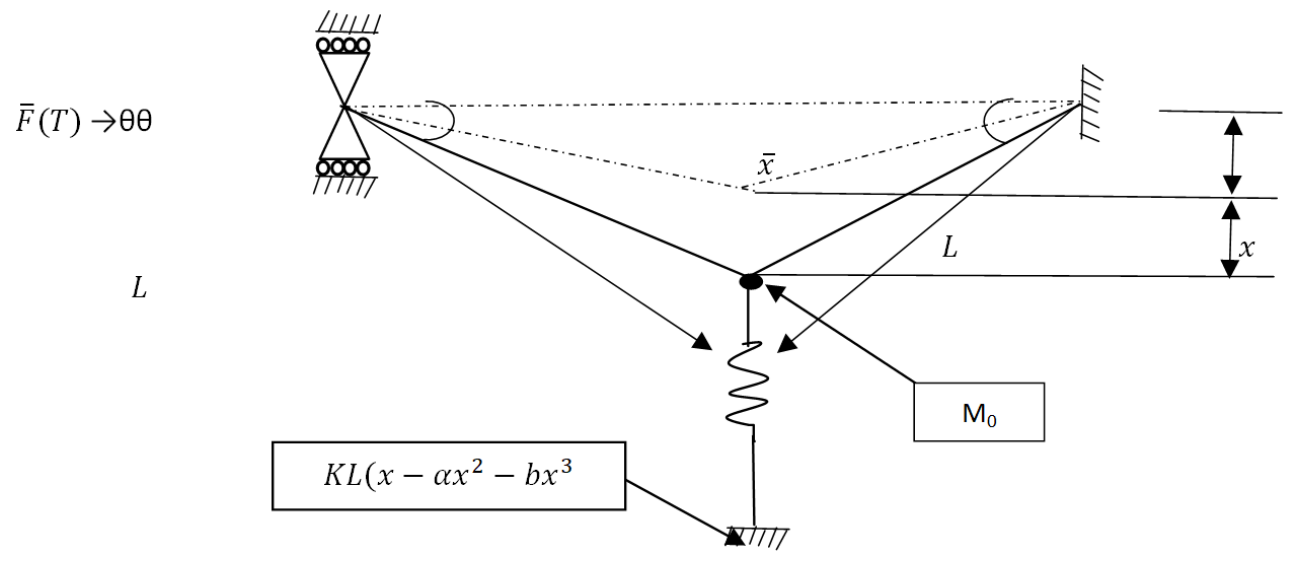

Figure 1. Simple quadratic-cubic model structure.

This study is an extension of the work of Budiansky's [28] as shown below:

$$
\ddot{\xi}+2 \bar{\xi} \dot{\xi}+(1-\lambda f(t)) \dot{\xi}+\alpha \xi^{2}-\beta \xi^{3}=\lambda f(t) \bar{\xi}, \quad t>0
$$




$$
\xi(0)=\dot{\xi}(0)=0, \quad 0<\bar{\xi}<1 .
$$

Here, $2 \bar{\xi}$ is the damping coefficient, $\lambda f(t)$ is a step load while $\lambda$ is a non-dimensional magnitude of the load satisfying the inequality $0<\lambda<1, f(t)$ is the actual load function which in our case is a step load satisfying $f(t)=0$ for $t>0$. $\alpha$ and $\beta$ are the imperfection sensitivity parameters. $\bar{\xi}$ is the amplitude of imperfection, $\xi$ is the deflection or displacement while $t$ is time parameter.

\section{Static Deformation at Imposition of the Pre-load $\lambda_{D}$}

Let the pre-static $\lambda_{0}$ be first impounded on the system and let $\xi_{0}$ be the time independent displacement at this level of loading. Then, from (2.1), it is clear that there is no damping. At this stage, $\lambda f(t) \equiv \lambda_{0}$ and the resultant equation is

$$
\left(1-\lambda_{0}\right) \xi_{0}+\alpha \xi_{0}^{2}-\beta \xi_{0}^{3}=\lambda_{0} \bar{\xi}
$$

where $\lambda_{0}$ is the static load.

By exploring the relative smallness of $\bar{\xi}$ relative to unity, the solution of (3.1) will be obtained using method of asymptotics by letting

$$
\xi_{0}=\sum_{i=1}^{\infty} \xi_{0}^{(i)} \bar{\xi}^{i} .
$$

According to Budiansky [28] and Hutchinson and Budiansky [32], the condition for maximum static buckling is

$$
\frac{d \lambda}{d \xi}=0
$$

The solution shall be made in two parts namely, the exact (phase plane) method and the approximate (asymptotic) method. The aim here is to compare the two methods. However, the asymptotic method will be generally more useful later when the solution involving the cylindrical shell is made. However problems involving cylindrical shell cannot be solved using the exact (phase plane) method, hence, the adoption of asymptotic procedures.

\subsection{Exact solution (Phase Plane Method)}

From (2.1), we have

$$
(1-\lambda) \xi+\alpha \xi^{2}-\beta \xi^{3}=\lambda \bar{\xi}, \quad 0<\bar{\xi}<1
$$


According to Budiansky [28] and, Hutchinson and Budiansky [32], the condition for static buckling is

$$
\frac{d \lambda}{d \xi_{s}}=0
$$

where $\xi_{s}$ is the displacement at buckling. Applying (3.4) on (3.1) at buckling, gives,

$$
(1-\lambda)+2 \alpha \xi_{s}-3 \beta \xi_{s}^{2}=0 .
$$

Solving (3.5), yields,

$$
\xi_{s}=\frac{2 \alpha \pm \sqrt{4 \alpha^{2}+12 \beta\left(1-\lambda_{s}\right)}}{6 \beta},
$$

where $\lambda_{s}$ is the static buckling load and,

$$
\xi_{s}=\alpha \pm\left(3 \beta\left(1-\lambda_{s}\right)\right)^{\frac{1}{2}}\left\{1+\frac{\alpha^{2}}{3 \beta\left(1-\lambda_{s}\right)}\right\}^{\frac{1}{2}}
$$

Further simplification of (3.7), gives,

$$
\xi_{s}=\frac{\alpha \pm\left(3 \beta\left(1-\lambda_{s}\right)\right)^{\frac{1}{2}} \varphi_{1}}{3 \beta},
$$

where

$$
\varphi_{1}=\left\{1+\frac{\alpha^{2} / \beta}{3\left(1-\lambda_{s}\right)}\right\}^{\frac{1}{2}} .
$$

Again simplifying (3.8) further yields,

$$
\xi_{s}=\frac{\left(3 \beta\left(1-\lambda_{s}\right)\right)^{\frac{1}{2}} \varphi_{2}}{3 \beta},
$$

where

$$
\varphi_{2}=\varphi_{1} \pm \frac{\alpha}{\left(3 \beta\left(1-\lambda_{s}\right)\right)^{\frac{1}{2}}} .
$$

Thus to obtain the static buckling load $\lambda_{s}$, we take the negative sign in (4.1) and let $\lambda=\lambda_{s}$. A further simplification of (4.0) gives, 


$$
\xi_{s}=\frac{\left(1-\lambda_{s}\right)^{\frac{1}{2}}}{(3 \beta)^{\frac{1}{2}}} \varphi_{2} .
$$

To determine the static buckling load, we determine (3.3) at static buckling, using (3.4), that is,

$$
\xi_{s}\left[\left(1-\lambda_{s}\right)+\alpha \xi_{s}-\beta \xi_{s}^{2}\right]=\lambda_{s} \bar{\xi}
$$

Multiplying (4.3) by 3, we get

$$
\xi_{s}\left[3\left(1-\lambda_{s}\right)+3 \alpha \xi_{s}-3 \beta \xi_{s}^{2}\right]=3 \lambda_{s} \bar{\xi}
$$

Substituting for $3 \beta \xi_{s}^{2}$ using (4.3), yields,

$$
\begin{gathered}
\xi_{s}\left[4\left(1-\lambda_{s}\right)+5 \alpha \xi_{s}\right]=3 \lambda_{s} \bar{\xi} \\
4\left(1-\lambda_{s}\right) \xi_{s}\left[1+\frac{5 \alpha}{4\left(1-\lambda_{s}\right)} \xi_{s}\right]=3 \lambda \bar{\xi}
\end{gathered}
$$

Substituting for $\xi_{s}$ using (4.2) and simplifying gives,

$$
\left(1-\lambda_{s}\right)^{\frac{3}{2}}=\frac{3 \sqrt{3} \lambda_{s} \xi \beta^{\frac{1}{2}}}{4 \varphi_{2}} \varphi_{3}
$$

where

$$
\varphi_{3}=\left[1+\frac{5 \alpha \varphi_{2}}{4 \sqrt{3 \beta}\left(1-\lambda_{s}\right)^{\frac{1}{2}}}\right]^{-1} .
$$

Equations (4.7) and (4.8) give the relevant formula for determining static buckling load, $\lambda_{s}$ and the method is exact as no approximation was involved in the solution.

\subsection{Asymptotic method}

Using (3.1) in (3.2a), we now obtain the following equations in powers of $\bar{\xi}$

$$
\begin{aligned}
& O(\bar{\xi}):\left(1-\lambda_{0}\right) \xi_{0}^{(1)}=\lambda_{0}, \\
& O\left(\bar{\xi}^{2}\right):\left(1-\lambda_{0}\right) \xi_{0}^{(2)}+\alpha \xi_{0}^{(1)^{2}}=0, \\
& O\left(\bar{\xi}^{3}\right):\left(1-\lambda_{0}\right) \xi_{0}^{(3)}+2 \alpha \xi_{0}^{(1)} \xi_{0}^{(2)}-\beta \xi_{0}^{(1)^{3}}=0 .
\end{aligned}
$$


It should be noted that $\xi_{0}^{(i)}$ is independent of time, $t$ and hence

$$
\frac{d}{d t}\left(\xi_{0}^{(i)}\right)=0
$$

To determine the static buckling of the model structure $\lambda_{s}$, we shall suppress the subscript in $\lambda_{0}$ as (3.1) and write $\lambda_{0}$ simply as $\lambda$ so that the relevant equation at this stage resulting from (3.1) is

$$
(1-\lambda) \xi+\alpha \xi^{2}-\beta \xi^{3}=\lambda \bar{\xi}, \quad 0<\bar{\xi}<1 .
$$

Hence equations (4.9) to (5.1) are now expressed in terms of $\lambda$ and not $\lambda_{0}$ and respectively take form,

$$
\begin{array}{r}
O(\xi):(1-\lambda) \xi^{(1)}=\lambda, \\
O(\xi):(1-\lambda) \xi^{(2)}+\alpha \xi^{(1)}=0, \\
O\left(\xi^{3}\right):(1-\lambda) \xi^{(3)}+2 \alpha \xi^{(1)} \xi^{(2)}-\beta \xi^{(1)^{3}}=0, \quad \text { etc. }
\end{array}
$$

Solving (5.4), gives

$$
\xi^{(1)}=\frac{\lambda}{1-\lambda}=B
$$

Similarly from (5.5) and (5.6), we obtain

$$
\begin{gathered}
\xi^{(2)}=-\frac{\alpha B}{1-\lambda}, \\
\xi^{(3)}=\frac{B^{3}}{1-\lambda}\left(\beta-\frac{2 \alpha^{2}}{1-\lambda}\right) .
\end{gathered}
$$

To determine the static buckling load, $\lambda_{s}$, we use $\lambda$ as the load parameter instead of $\lambda_{0}$ in (5.7), (5.8) and (5.9).

The condition for static buckling is

$$
\frac{d \lambda}{d \xi}=0
$$

The displacement, $\xi_{0}$ is given as

$$
\xi(\lambda)=\xi^{(1)} \bar{\xi}+\xi^{(2)} \bar{\xi}^{2}+\xi^{(3)} \bar{\xi}^{3}+\cdots .
$$


We can rewrite (6.1) as

$$
\xi=C_{1} \bar{\xi}+C_{2} \bar{\xi}^{2}+C_{3} \bar{\xi}^{3}+\cdots,
$$

where

$$
C_{1}=\xi^{(1)}, C_{2}=\xi^{(2)}, C_{3}=\xi^{(3)} .
$$

According to Ette [30], we first reverse the series (6.2) in the form

$$
\bar{\xi}=d_{1} \xi+d_{2} \xi^{2}+d_{3} \xi^{3}+\cdots .
$$

Substituting $\xi$ from (6.2) in (6.3) and equate the coefficient of powers of $\bar{\xi}$, we get,

$$
\begin{array}{r}
O(\bar{\xi}): 1=d_{1} C_{1}, \\
O\left(\bar{\xi}^{2}\right): 0=d_{1} C_{1}+d_{2} C_{1}^{2}, \\
O\left(\bar{\xi}^{3}\right): 0=d_{1} C_{3}+2 d_{2} C_{1} C_{2}+d_{3} C_{1}^{3} .
\end{array}
$$

Solving $(6.4 \mathrm{a}, \mathrm{b}, \mathrm{c})$, gives

$$
d_{1}=\frac{1}{C_{1}} ; d_{2}=-\frac{C_{3}}{C_{1}^{3}} \quad ; d_{3}=\frac{2 C_{2}^{2}-C_{1} C_{3}}{C_{1}^{5}} .
$$

To determine the static buckling load, $\lambda_{s}$, we use (6.0) on (6.3) and get

$$
\begin{gathered}
\frac{d \lambda}{d \xi}=\left(\frac{d d_{1}}{d \lambda} \frac{d \lambda}{d \xi}+d_{1}\right)+\left(\frac{d d_{2}}{d \lambda} \frac{d \lambda}{d \xi}+2 d_{2} \xi\right)+\left(\frac{d d_{3}}{d \lambda} \frac{d \lambda}{d \xi}+3 d_{3} \xi^{2}\right)+\cdots=0 \\
\therefore d_{1}+2 d_{2} \xi_{s}+3 d_{3} \xi_{s}^{2}=0
\end{gathered}
$$

where $\xi_{s}$ is the value of $\xi$ at static buckling

$$
\therefore \quad \xi_{s}=\frac{-2 d_{1} \pm \sqrt{4 d_{2}^{2}-12 d_{1} d_{3}}}{6 d_{3}} .
$$

Taking the negative square root of (6.7b) and simplifying, yields

$$
\xi_{s}=\frac{-d_{1}-\sqrt{d_{2}^{2}-3 d_{1} d_{3}}}{3 d_{3}} .
$$

Further simplification gives,

$$
\xi_{s}=\left(\frac{1}{3 d_{3}}\right)\left[-d_{2}-\left(d_{2}^{2}-3 d_{1} d_{3}\right)^{\frac{1}{2}}\right] .
$$


Substituting the values of $d$ and simplifying, gives

$$
\xi_{s}=\left(\frac{1-\lambda_{s}}{3 \beta \varphi_{0}}\right)^{\frac{1}{2}}\left(\frac{Q_{1}^{\frac{1}{2}}}{Q_{2}}\right)\left[1-\frac{B^{3}\left(\alpha / \beta^{\frac{1}{2}}\right)}{\sqrt{3 \varphi_{0}\left(1-\lambda_{s}\right)}}\right],
$$

where

$$
\begin{gathered}
Q_{1}=1-\frac{5\left(\frac{\alpha^{2}}{\beta}\right)}{3\left(1-\lambda_{s}\right) \varphi_{0}} \\
\varphi_{0}=1+\frac{\left(\frac{2 \alpha^{2}}{\beta}\right)}{1-\lambda_{s}} \\
Q_{2}=1-\frac{\left(\frac{2 \alpha^{2}}{\beta}\right)}{\left(1-\lambda_{s}\right) \varphi_{0}} .
\end{gathered}
$$

The static buckling load, $\lambda_{s}$, is determined by evaluating (6.3) at static buckling to get

$$
\bar{\xi}=\xi_{s}\left(d_{1}+d_{2} \xi_{s}+d_{3} \xi_{s}^{2}\right)+\cdots .
$$

Multiplying (6.9a) by 3, noting that $3 d_{3} \xi_{s}^{2}=-d_{1}-2 d_{2} \xi_{s}$ and using (6.7a), then (6.9a) becomes

$$
3 \bar{\xi}=\xi\left(2 d_{1}+d_{2} \xi_{s}\right)
$$

Substituting for $d$ and $\xi_{s}$ in (6.9b) and on simplification, we get

$$
\left(1-\lambda_{s}\right)^{\frac{3}{2}}=\frac{3 \sqrt{3}}{2}\left(\lambda_{s} \xi\right)\left(\beta \varphi_{0}\right)^{\frac{1}{2}}\left(\frac{Q_{2}}{Q_{1}^{\frac{1}{2}}}\right)\left[1-\frac{B^{3}\left(\alpha / \beta^{\frac{1}{2}}\right)}{\sqrt{3}\left(1-\lambda_{s}\right)^{\frac{1}{2}}}\right]^{-1} \times\left[1-\frac{\alpha \xi_{s}}{2\left(1-\lambda_{s}\right)}\right]^{-1},
$$

where $\lambda_{s}$ is the static buckling load of the model structure. Equation (7.0) gives an implicit formula for obtaining the static buckling load $\lambda_{s}$ and the result is asymptotic in nature. 


\section{Analysis and Discussion}

Table 1. Comparison of the exact and asymptotic methods.

\begin{tabular}{|c|c|c|}
\hline $\begin{array}{c}\text { Imperfection } \\
\text { Parameter } \bar{\xi}\end{array}$ & Exact Results & Asymptotic Results \\
\hline 0.01 & 0.776304 & 0.463453 \\
\hline 0.02 & 0.732976 & 0.411338 \\
\hline 0.03 & 0.704091 & 0.379317 \\
\hline 0.04 & 0.681894 & 0.356100 \\
\hline 0.05 & 0.663665 & 0.337699 \\
\hline 0.06 & 0.648102 & 0.322629 \\
\hline 0.07 & 0.634466 & 0.309792 \\
\hline 0.08 & 0.622299 & 0.298646 \\
\hline 0.09 & 0.611294 & 0.288794 \\
\hline 0.10 & 0.601231 & 0.279976 \\
\hline
\end{tabular}

\section{Static buckling load against imperfection}

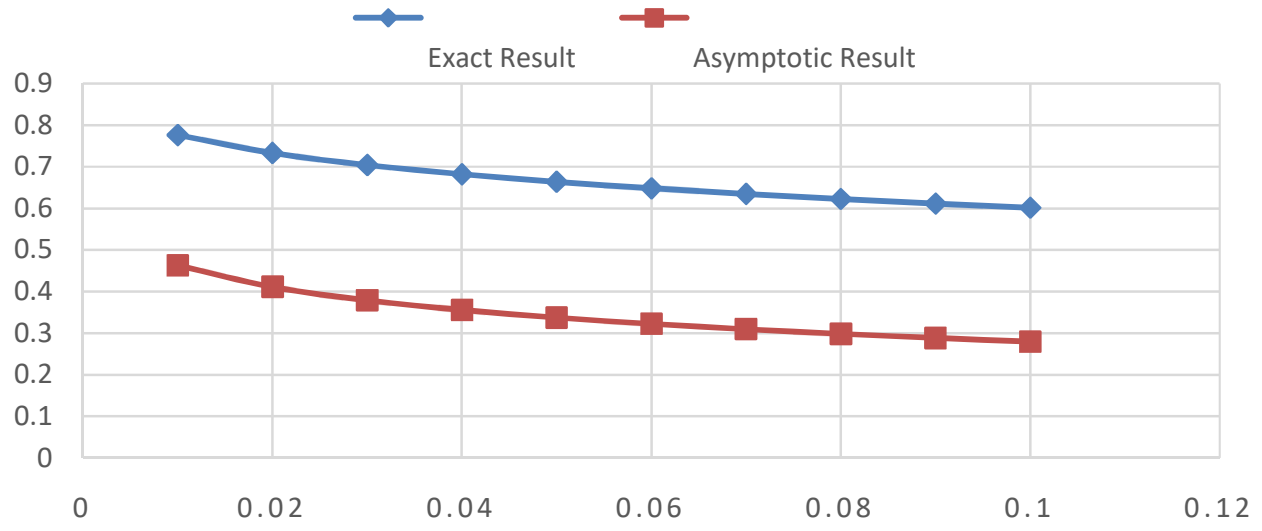

Figure 2. Graphical plot showing the comparison of the phase plane method and the method of asymptotics.

From the results obtained from both Table 1 and Figure 2, we observe the following:

a. The static buckling loads obtained using the phase plane (exact method) are higher than the static buckling loads obtained using asymptotic method. 
b. The static buckling loads decrease as the imperfection parameters in the structure increase. This result agrees with the results in the existing literatures.

Most physical systems are represented using differential equations and many of such physical systems are coupled and nonlinear in nature and cannot be solved using exact method, so it is our contention that such nonlinear systems will be analyzed using the method of asymptotics.

\section{References}

[1] H. E. Lindberg, Little Book of Dynamic Buckling, LCE Science/Software, 2003; [Online]. Available from: www.lindbergice.com/tech/buklbook.htm

[2] Z. Kołakowski, Static and dynamic interactive buckling of composite columns, Journal of Theoretical and Applied Mechanics 47(1) (2009), 177-192.

[3] V. Chitra and R. S. Priyadarsini, Dynamic buckling of composite cylindrical shells subjected to axial impulse, International Journal of Scientific \& Engineering Research 4(5) (2013), 162-165.

[4] G. J. Simitses, Effect of static preloading on the dynamic stability of structures, AIAA Journal 21(8) (1983), 1174-1180. https://doi.org/10.2514/3.8223

[5] A. Tabiei, R. Tanov and G. J. Simitses, Numerical simulation of cylindrical laminated shells under impulsive lateral pressure, AIAA Journal 37 (1999), 629-633. https://doi.org/10.2514/2.763

[6] R. Tanov, A. Tabiei and G. J. Simitses, Effect of static preloading on the dynamic buckling of laminated cylinders under sudden pressure, Mechanics of Composite Materials and Structures 6 (1999), 195-206. https://doi.org/10.1080/107594199305520

[7] M. Jabareen and S. Izhak, Dynamic buckling of a beam on a nonlinear elastic foundation under step loading, Journal of Mechanics of Materials and Structures 4(7-8) (2009), 1365-1374. https://doi.org/10.2140/jomms.2009.4.1365

[8] J. Jankowski, Buckling and vibrations of composite column-beams, Stability of Structures XIII-th Symposium - Zakopane (2012), 289-294.

[9] A. Patil, A. Kolhe and Abdul Sayeed A. W. Shaikh, Review of buckling in various structures like plate \& shells, International Journal of Research in Engineering and Technology 03(04) (2014), 396-402. https://doi.org/10.15623/ijret.2014.0304072

[10] M. S. Qatu, Ebrahim Asadi and Wenchao Wang, Review of recent literature on static 
analyses of composite shells: 2000-2010, Open Journal of Composite Materials 2 (2012), 61-86. https://doi.org/10.4236/ojcm.2012.23009

[11] M. Touati, A. Chelghoum and R. C. Barros, Numerical methods for determining the dynamic buckling critical load of thin shells: state of the art, Buletinul Institutului Politehnic Din Iaşi Publicat de Universitatea Tehnică "Gheorghe Asachi” din Iaşi Tomul LVIII (LXII) 1 (2012), 21-36.

[12] G. J. Simitses, Buckling and postbuckling of imperfect cylindrical shells: a review, Appl. Mech. Rev. 39(10) (1986), 1517-1524. https://doi.org/10.1115/1.3149506

[13] S. K. Sahu and P. K. Datta, Research advances in the dynamic stability behaviour of plates and shells: 1987-2005-Part 1: conservative systems, Applied Mechanics Review 60 (2007), 66-75. https://doi.org/10.1115/1.2515580

[14] D. Y. Jeong, Analyses for lateral deflection of railroad track under quasi-static loading, Proceedings of the ASME 2013 Rail Transportation Division Fall Technical Conference, 2013, 10 pp. https://doi.org/10.1115/RTDF2013-4710

[15] B. P. Russell, S. D. Vikram and N. G. W. Haydn, Quasi-static deformation and failure modes of composite square honeycombs, Journal of Mechanics of Materials and Structures 3(7) (2008), 1315-1340. https://doi.org/10.2140/jomms.2008.3.1315

[16] H. Zareiforoush, H. Mohammad, Z. Komari and R. A. Mohammad, Mechanical properties of paddy grains under quasi-static compressive loading, New York Science Journal 3(7) (2010), 40-46.

[17] E. Eglitis, K. Kalnins, O. Ozolins and R. Rikards, Numerical study of geometrical imperfections response on composite cylinders under axial load, Proceedings of 20th Nordic Seminar on Computational Mechanics, Gothenburg, Sweden, 2007, pp. 101-104.

[18] T. Lu and T. J. Wang, Asymptotic solutions for buckling delamination induced crack propagation in the thin film-compliant substrate system, Theoretical and Applied Mechanics Letters 4(4) (2014), 041003.

[19] R. Lewandowski, Analysis of strongly non-linear free vibrations of beams using perturbation method, Civil and Environmental Engineering Reports 1 (2005), 153-167.

[20] S. Reboux, G. Richardson and O. Jensen, An asymptotic analysis of the buckling of a highly shear-resistant vesicle, Euro. Jnl of Applied Mathematics 20 (2009), 479-518. https://doi.org/10.1017/S0956792509990015

[21] B. Eirik, S. Eivind and A. Jørgen, A semi-analytical model for global buckling and postbuckling analysis of stiffened panels, Thin-Walled Structures 42 (2004), 701-717. https://doi.org/10.1016/j.tws.2003.12.006 
[22] J. C. Amazigo, B. Budiansky and G. F. Carrier, Asymptotic analyses of the buckling of imperfect columns on nonlinear elastic foundations, Int. J. Solids Structures 6(10) (1970), 1341-1356. https://doi.org/10.1016/0020-7683(70)90067-3

[23] H. Qiang, S. Zhang and G. Yang, The asymptotic solution of a dynamic buckling problem in elastic columns, Applied Mathematics and Mechanics 20(8) (1999), 867-872. https://doi.org/10.1007/BF02452484

[24] A. M. Ette, Dynamic buckling of an imperfect spherical shell under an axial impulse, Int. J. Non-Linear Mech. 32 (1997), 201-209. https://doi.org/10.1016/S0020-7462(96)00051-0

[25] A. M. Ette, On a two-parameter dynamic buckling of a lightly damped spherical cap trapped by a step load, J. Nigerian Math. Soc. 23 (2004), 7-26.

[26] A. M. Ette and J. U. Onwuchekwa, On the static buckling of an externally pressurized finite circular cylindrical shell, Journal of the Nigerian Association of Mathematical Physics 11 (2007), 323-332. https://doi.org/10.4314/jonamp.v11i1.40226

[27] C. M. Bender and S. A. Orszag, Advanced Mathematical Methods for Scientists and Engineers I-Asymptotic Methods and Perturbation Theory, Springer, 1999. https://doi.org/10.1007/978-1-4757-3069-2

[28] B. Budiansky, Dynamic buckling of elastic structures: criteria and estimates, in: Dynamic Stabilities of Structures, G. Herrmann, ed., Pergamon, Oxford, 1967, pp. 83-106. https://doi.org/10.1016/B978-1-4831-9821-7.50010-7

[29] D. Danielson, Dynamic buckling loads of imperfection-sensitive structures from perturbation procedures, AIAA Journal 7 (1969), 1506-1510. https://doi.org/10.2514/3.5423

[30] A. M. Ette, On a lightly damped elastic quadratic model structure modulated by a dynamic periodic load, J. Nigerian Assoc. Math. Phys. 14 (2009), 21-40.

[31] Joy U. Chukwuchekwa and Anthony M. Ette, Asymptotic analysis of an improved quadratic model structure subjected to static loading, J. Nigerian Assoc. Math. Phys. 32 (2015), 237-244.

[32] J. W. Hutchinson and B. Budiansky, Dynamic buckling estimates, AIAA Journal 4(3) (1966), 525-530. https://doi.org/10.2514/3.3468

\footnotetext{
This is an open access article distributed under the terms of the Creative Commons Attribution License (http://creativecommons.org/licenses/by/4.0/), which permits unrestricted, use, distribution and reproduction in any medium, or format for any purpose, even commercially provided the work is properly cited.
} 